\title{
Aplicación de métodos cuantitativos en la programación de eventos: una aplicación en el carnaval uruguayo
}

Application of quantitative methods for the programming of artistic shows: an application in the uruguayan carnival

\author{
Alfonsina Cardozo', Carolina Guido², Juan Carlos Machin ${ }^{3}$
}

Recibido: Febrero 2021

Aceptado: Marzo 2021

Resumen.- En este trabajo se presenta una aplicación de métodos cuantitativos para la programación de espectáculos artísticos. Específicamente, el trabajo realizado para el problema de la confección del fixture del Concurso de Carnaval de las Promesas 2019/2020. Dicho evento, es una competencia perteneciente al carnaval uruguayo que cuenta con cinco categorías artísticas diferentes, dirigida a agrupaciones conformadas por niños y adolescentes. Se desarrolló un modelo de programación matemática entera mixta para el problema, con el objetivo de equilibrar la asistencia de público y cumplir con las restricciones del reglamento del concurso, así como con otros requerimientos indicados por los organizadores.

Palabras clave: Organización de Eventos Artísticos; Toma de decisiones; Programación matemática; Timetabling; Optimización.

Summary.- In this work an application of quantitative methods for the programming of artistic shows is presented. In particular, the work carried out for the problem of making the fixture for the contest "Carnaval de las Promesas 2019/2020". This event is a competition belonging to the Uruguayan carnival that has five different artistic categories, created especially for groups of children and adolescents. A mixed integer mathematical programming model was developed for the problem. The objective was to balance the attendance of the public and comply with the restrictions of the contest regulations, as well as other requirements indicated by the organizers.

Keywords: Organization of Artistic Events; Decision Making; Mathematical Programming; Timetabling; Optimization.

\footnotetext{
${ }^{1}$ Ingeniera de Producción, Facultad de Ingeniería, Universidad de la República, alfoncardozo@gmail.com, ORCID iD: https://orcid.org/0000-0001-6507-2281

2 Ingeniera de Producción, Facultad de Ingeniería, Universidad de la República, guica_11@hotmail.com, ORCID iD: https://orcid.org/0000-0003-0271-830X

3 Ingeniero de Producción, Facultad de Ingeniería, Universidad de la República, jmachin@fing.edu.uy, ORCID iD: 0000- https://orcid.org/0002-4564-6089
} 
Introducción.- El motivo por el que se decide llevar a cabo este trabajo fue el desafío que implicaba confeccionar la programación de los espectáculos teniendo en cuenta ciertas restricciones, asignando para un conjunto de espacios de tiempo, el conjunto de espectáculos disponibles. Dicha asignación suele realizarse según el conocimiento de una persona competente o al azar [1]. Esto implica algunos inconvenientes, como puede ser no tener presente todas las consideraciones de las partes interesadas [2]. Por eso, se considera de interés generar una herramienta para la confección del calendario de forma objetiva y teniendo en cuenta las preferencias de las diferentes partes interesadas.

Para definir un evento, se toma la definición de la Real Academia Española [3], en la cual un evento es un "suceso importante y programado, de índole social, académica, artística o deportiva". Se llama evento entonces a un suceso programable, que ocurre en un espacio de tiempo determinado. Un conjunto de eventos es un acontecimiento que puede ser de índole social, académico, artístico o deportivo. Dentro del acontecimiento se le asigna a cada evento un espacio de tiempo.

Dado entonces un conjunto acotado de eventos, se denomina programación de eventos al proceso de asignar cada uno de los eventos a un cierto espacio de tiempo, con un inicio y un fin, de un conjunto finito de espacios de tiempo disponibles. El objetivo es organizar las actividades teniendo en cuenta las necesidades y preferencias de las diferentes partes interesadas. De esta manera se logra la satisfacción de estos y en la mayoría de los casos mayor rentabilidad para el evento [4]. Por partes interesadas se refiere a cualquier individuo, grupo u organización que forme parte o se vea afectado por el mismo, obteniendo algún beneficio o perjuicio.

El primer objetivo de este proyecto consistió en realizar un relevamiento sobre la bibliografía en torno a la aplicación de métodos cuantitativos en la programación de eventos, con el fin de mostrar en qué fase de desarrollo se encuentra este tema. Como resultado del relevamiento se realizó un estado del arte, con un abordaje sobre métodos cuantitativos aplicados a problemas de programación de un acontecimiento y problemas de asignación relacionados.

El segundo objetivo del proyecto fue tomar un caso de estudio de un espectáculo artístico local en donde sea relevante realizar la programación del calendario de ese acontecimiento. El espectáculo seleccionado fue el Carnaval de las Promesas, un concurso donde participan alrededor de 2.000 jóvenes todos los años. Dicho concurso es desarrollado por la Intendencia de Montevideo en coordinación con la Asociación de Directores del Carnaval de las Promesas (ADICAPRO), una institución sin fines de lucro que tiene como objetivo el desarrollo artístico, personal y cultural de niños y adolescentes.

Obtenido el caso de estudio, se desarrolló en primer lugar un modelo matemático de programación del calendario. Para determinar el modelo, se estudió el reglamento del concurso, así como también fueron consultados expertos del tema, particularmente las personas que confeccionan actualmente el calendario. Para la recolección y análisis de los datos necesarios, se contó con el apoyo de ADICAPRO y se realizaron encuestas a todas las agrupaciones participantes de dicho concurso. El objetivo es determinar la programación con concurrencia equilibrada y que cumpla con las restricciones del reglamento y otras restricciones que permiten una programación más atractiva para el público y más justa para las agrupaciones.

1. Aplicación de métodos cuantitativos en la confección de calendarios.- La utilización de modelos matemáticos permite la comprensión y la resolución de problemas, los cuales implican abstracciones que tienen en cuenta las interacciones relevantes de las entidades de dicho problema. Se busca con el modelo determinar una solución óptima dentro de un conjunto factible de decisiones. Los diferentes trabajos relevados en la confección de nuestro estado del arte se clasifican en dos tipos: problemas de programación de un acontecimiento y problemas de asignación relacionados. A su vez, dentro de los primeros, se clasifican según el tipo de evento: deportivo, académico o artístico.

Como conclusión de dicha investigación observamos que la menor cantidad de trabajos fue 
encontrada en el campo artístico, donde dentro del mismo la mayor parte son sobre programación de comerciales de TV, y solamente tres artículos sobre el tema de motivación de nuestro estudio. La categoría de trabajos deportivos es donde encontramos la mayor cantidad de trabajos dentro de programación de eventos. Encontramos que los métodos cuantitativos han sido aplicados a diferentes deportes, con el foco, en la mayoría de los casos, de maximizar ganancias. Por otro lado, en el campo académico, los trabajos se centran en beneficiar a las partes interesadas. Por último, en la categoría de problemas de tipo asignación fue donde se encontró la mayor cantidad de trabajos, a pesar de no estar contenidos en nuestra definición de programación de eventos, pero que a nuestra consideración aportan a la comprensión del tema.

En la mayoría de los trabajos encontrados los métodos cuantitativos son una herramienta que permite obtener una solución de mejor calidad. Esta solución obtenida es una herramienta de ayuda en la toma de decisión. Identificamos una falta de bibliografía específica sobre la programación de eventos artísticos.

2. Carnaval uruguayo.- El Carnaval en Uruguay es una fiesta popular de carácter internacional. Se lo declaró de Interés Nacional, se lo considera como la máxima fiesta popular y es el carnaval más largo del mundo. Empieza a finales de enero y continúa hasta mediados de marzo, durante más de 50 días, consistiendo en desfiles callejeros y escenarios barriales. Con el fin de seleccionar como caso de estudio un espectáculo artístico nacional en donde se realice la programación del evento, se trabajó con el carnaval de las promesas.

El carnaval tiene una fuerte relevancia en la cultura uruguaya. Esto se ve principalmente con el Concurso Oficial de Carnaval, que es excluyente para mayores de edad. Con el objetivo de promover la inclusión social y la integración de niñas, niños y adolescentes y fomentar el desarrollo artístico, personal y cultural de los mismos surge el Carnaval de las Promesas.

El Carnaval de las Promesas es organizado por el Departamento de Cultura de la Intendencia de Montevideo en coordinación con la Asociación de Directores del Carnaval de las Promesas (ADICAPRO).

Mediante el carnaval de las promesas se pretende que los niños logren un desarrollo personal y artístico, que logrará que, en futuras etapas de sus vidas, consigan tener una visión del mundo y las personas que los rodean, que les sea útil para alcanzar sus metas personales y profesionales. En la actualidad participan del concurso entre 1500 y 2000 niños y jóvenes entre los 5 y 18 años. Durante el año se apunta a realizar una actividad cultural con talleres de música, canto, coreografía, baile, actuación, maquillaje, vestuario y escenografía. Esto es llevado a cabo por niños, padres, excomponentes y técnicos formados dentro y fuera del Carnaval de las Promesas.

3. Análisis y resolución del problema.- Para entender el Carnaval de las Promesas y el proceso actual de programación, se consultó a los integrantes de ADICAPRO y la Gerencia de Eventos de la Intendencia de Montevideo, donde vimos la modalidad del concurso, las agrupaciones participantes, los tipo de agrupaciones y cómo confeccionaban el calendario los años anteriores. Más allá de encontrar una solución, la participación de los encargados actuales en la elección y validación del calendario es imprescindible para que éste sea acorde a sus criterios. La herramienta brindada es un apoyo a la toma de decisiones de la dirección, que no sustituye al responsable de tomar las decisiones.

Al profundizar en el tema, se observa que la elaboración del calendario por el método manual utilizado actualmente implica varias jornadas de trabajo. Además de ser complejo tener en cuenta todo el reglamento [5], también es posible que al realizar modificaciones causen un desajuste en otro aspecto. Por ello, es beneficioso encontrar un modelo matemático que represente correctamente la realidad, teniendo en cuenta todas las restricciones del sistema y que permita encontrar una solución en un menor tiempo. 
3.1. Modelo matemático.- El objetivo es lograr una programación del calendario del Carnaval de las Promesas de manera que las etapas sean equilibradas en relación con la asistencia del público. Para esto, se minimiza las desviaciones de la ponderación de popularidad de cada etapa con respecto al promedio de ponderaciones

La manera optada para medir la concurrencia de las agrupaciones fue mediante el uso de encuestas. Para involucrar a las agrupaciones en este proyecto las encuestas fueron realizadas por ellos, lo cual también lleva a motivarlos en su entendimiento y aceptación del nuevo método de creación del fixture. De esta manera, un delegado de cada agrupación debió indicar si la convocatoria del resto de las agrupaciones era alta, mediana o baja. Se prefirió que la agrupación no puntúe a otras de su misma categoría para lograr una mayor objetividad.

Los parámetros de entrada del modelo están compuestos por las agrupaciones a programar, su respectiva ponderación de popularidad, el promedio de esta ponderación, agrupaciones que cuentan con bandas y los conjuntos de agrupaciones que pueden cerrar y abrir una etapa.

En la edición del concurso 2019/2020 participaron 40 agrupaciones. El concurso se desarrolló en 2 rondas de 10 etapas cada una. El modelo matemático creado permite resolver las dos rondas al mismo tiempo, buscando el óptimo global y teniendo en cuenta las restricciones que relacionan las dos rondas.

3.2. Resolución.- El modelo cuenta con 37 familias de restricciones, para corroborar el funcionamiento del modelo, se asignó arbitrariamente valores a los parámetros del sistema: ponderaciones de las agrupaciones y promedio. Se incluyeron gradualmente diferentes restricciones de manera de ir validando el modelo de a grupos de restricciones y procurando obtener una solución en cada caso.

Se decide usar para todo el proyecto el paquete de software de optimización AMPL-CPLEX versión 12.8.0.0 y versión 12.9.0.0. Se corrieron los modelos en la computadora de características: CPU Intel Core i7 -6700 -3.40GHz -24GB RAM.

Para determinar el calendario para las dos rondas del concurso, se obtuvo una solución con un tiempo de procesamiento de aproximadamente 14000 segundos.

3.3. Resolución alternativa.- Se analizó la posibilidad de resolver el problema mediante otra estrategia de resolución. La misma consiste en resolver ambas rondas del concurso de manera independiente, mediante dos modelos distintos. En una primera instancia, se resuelve la ronda 1 y con la información obtenida, se resuelve en otro modelo la ronda 2.

Esta metodología implica dos modelos diferentes, derivados de modificaciones del modelo original utilizado para calcular las dos rondas. En el Modelo Ronda 1, se eliminan las restricciones que involucran a la ronda 2 , y se obtiene una programación únicamente para la ronda 1 del concurso. Mientras que en el Modelo Ronda 2, se ingresa la solución del Modelo Ronda 1 como parámetros del modelo, para poder obtener una ronda 2 que tenga en cuenta las asignaciones de agrupaciones de la ronda 1. También se eliminan de este último modelo aquellas restricciones que involucran solo a la ronda 1 .

Con la resolución alternativa concluimos que no siempre se podrá utilizar, porque con los datos de la ronda 1, la ronda 2 puede ser no factible, dependerá de los datos, consideraciones y parámetros del caso de estudio.

Si bien esta metodología implica una disminución del tiempo de procesamiento de la ronda 1 con respecto al modelo de ambas rondas juntas, no asegura encontrar solución para la ronda 2 . Cuando nos planteamos resolver las dos rondas al mismo tiempo, el programa busca el óptimo para ambas rondas teniendo en cuenta las restricciones que las relacionan. Al eliminar estas restricciones y no buscar el óptimo global, puede que no se encuentre una solución factible para la ronda dos.

Por esta razón decidimos descartar la alternativa de resolver el problema en dos etapas, utilizando el modelo base para determinar la programación de ambas rondas. 
Sin embargo, un modelo que permita programar la ronda 2 con datos de la ronda 1, es una herramienta valorada para ADICAPRO. Esto se debe a que hay cierta probabilidad de que alguna etapa de la primera ronda se suspenda, por ejemplo, a causa de inclemencias del clima. Esta estrategia permite tener en cuenta lo que sucedió en la primera ronda para poder programar la siguiente ronda.

4. Experimentación numérica.- El objetivo de la experimentación numérica es evaluar el resultado obtenido en diferentes casos de prueba, considerando el tiempo de procesamiento, el valor de la función objetivo y los calendarios obtenidos. Para llevar a cabo la experimentación numérica se plantearon diferentes casos de prueba.

En base al modelo de resolución de ambas rondas, se realizó una experimentación numérica donde se evalúa el impacto en el calendario resultante al modificar parámetros de entrada. Esto es necesario dado que los datos pueden tener cierta incertidumbre y es útil saber cómo esto se refleja en la solución correspondiente. A partir de esta evaluación surge que el calendario resultante depende de los datos ingresados, por lo cual es importante el tratamiento de las encuestas de manera de que estos datos sean lo más representativos de la realidad como sea posible.

También con base en el modelo de resolución de ambas rondas, con el objetivo de determinar el impacto que tiene inicializar parcialmente información en el solver, se decide estudiar cómo este comporta computacionalmente al inicializar distintas variables de decisión. Para ello, se fija el tiempo de procesamiento y se evalúa el valor de la función objetivo obtenido en diferentes pruebas al inicializar distintas variables. De esto, se puede concluir que inicializar variables no aporta a tener una solución de mejor calidad.

Finalmente, se evaluaron diferentes situaciones en caso de que ocurran suspensiones de alguna etapa de la ronda 1 . Si alguna etapa de la ronda 1 es suspendida durante el concurso, el calendario para la segunda ronda debe reprogramarse teniendo en cuenta lo acontecido en la ronda anterior. En ciertos casos analizados se eliminaron algunas restricciones para lograr factibilidad. Se destaca que, en caso de que exista suspensión de alguna o varias etapas, el reprogramar el calendario de la segunda ronda del concurso con los datos de la ronda 1 como entrada implica un tiempo de procesamiento en cuestión de segundos. Esto favorece a ADICAPRO porque los días entre la ronda 1 y ronda 2 son acotados y se debe presentar la programación de la ronda 2 rápidamente, para que las agrupaciones planifiquen su presentación. Al suspender etapas y reprogramar el calendario, el valor de la función objetivo no mejora con respecto al caso base, ya que la solución en este caso es más restringida. Asimismo, no hay una relación entre la cantidad de etapas que se suspendieron y los calendarios obtenidos. Dependiendo de la etapa que se suspendió, varía como afecta al calendario resultante.

5. Conclusiones.- Como primera conclusión, afirmamos que se alcanzó el primer objetivo del proyecto, el cual consistió en realizar un relevamiento sobre métodos cuantitativos aplicados a la programación de eventos, y más específicamente los vinculados a espectáculos artísticos.

Con respecto al segundo objetivo, el cual consistió en aplicar esta metodología a un evento cultural local, se trabajó junto a ADICAPRO y la Intendencia de Montevideo, para determinarla programación del Concurso de las Promesas 2019/2020. Este objetivo también fue alcanzado ya que el calendario obtenido, luego de unas modificaciones manuales, fue utilizado en la edición del concurso 2019/2020 [6].

Consideramos enriquecedor el aplicar la ingeniería a temas de la cultura local, donde participan una gran cantidad de jóvenes. Como estudiantes de la Universidad de la República, poder volcar lo que aprendimos a la sociedad nos llena de orgullo y emoción. También, consideramos que presentar este trabajo a jóvenes puede motivar la participación de estos en una carrera universitaria científica-tecnológica y demostrar cómo esta puede relacionarse con las áreas culturales.

Confeccionar el calendario mediante esta herramienta es una manera objetiva, que cuenta con un

Memoria Investigaciones en Ingeniería, núm. 20 (2021). pp 16-23

https://doi.org/10.36561/ING.20.3

ISSN 2301-1092•ISSN (en línea) 2301-1106 
respaldo matemático, a diferencia de los sorteos utilizados actualmente por ADICAPRO. Si bien los sorteos son una manera imparcial para el armado, no se tiene en cuenta la ventaja de encontrar un calendario óptimo según un criterio definido. Se logró obtener un calendario factible con concurrencia equilibrada y que cumpla con las restricciones del reglamento y las consideraciones más justas para todas las agrupaciones. Otra ventaja respecto a la metodología manual es reducir el tiempo de armado del calendario. Al aplicar métodos cuantitativos se obtienen distintos calendarios tomando en cuenta diferentes consideraciones. Esto le permite al tomador de decisiones poder compararlos y elegir el calendario que mejor se adapten a sus necesidades.

Luego de relevado el caso de estudio se elaboró un modelo matemático para resolver las dos rondas del concurso simultáneamente, teniendo en cuenta las relaciones determinadas en el reglamento del concurso y lo indicado por la organización. La función objetivo del modelo fue encontrar una programación del concurso de manera que las etapas sean equilibradas en relación con la asistencia del público. Los datos para el modelo fueron obtenidos mediante encuestas realizadas a las diferentes agrupaciones. Vale destacar, que el involucramiento de los participantes en las encuestas de convocatoria de las agrupaciones lleva a que estos se sientan parte de la creación del calendario. Esto genera un acercamiento con los integrantes, logrando que se acepte nuestro trabajo de forma más sencilla. Además, al analizar la experimentación numérica observamos la importancia de estos datos en el calendario final, por eso se procura que esta información refleja la realidad de la convocatoria.

Por otro lado, se analizó la posibilidad de resolver el problema mediante otra estrategia de resolución. La misma consiste en resolver ambas rondas de manera independiente, mediante dos modelos distintos. En una primera instancia, se resuelve la ronda 1 y con la información obtenida, se resuelve en otro modelo la ronda 2.

En un principio creímos que resolverlas por separado no generaba valor, ya que consideramos pertinente encontrar un óptimo global para ambas rondas del concurso mediante el modelo de ambas rondas. Luego a lo largo del transcurso del carnaval, observando la cantidad de suspensiones de etapas que ocurrieron, concluimos que, dada la alta probabilidad de inclemencias climáticas, no permite utilizar la ronda 2 obtenida originalmente con el modelo de ambas rondas. Entendimos la importancia de contar con un modelo que encuentre el óptimo para la ronda 2 con los datos de lo acontecido en ronda 1.

Esto también nos lleva a pensar que quizás es conveniente obtener la programación de la ronda 1 con el Modelo Ronda 1, y luego con los datos de la realidad utilizando el Modelo Ronda 2, hallar la programación para la ronda siguiente. En el caso de que haya suspensiones en la ronda 1, de cualquier manera, se utiliza el Modelo Ronda 2. Esta metodología implica un mejor valor de función objetivo para la ronda 1 y una reducción en el tiempo de procesamiento para la obtención de los calendarios. Por lo tanto, basándose en la probabilidad de que existan suspensiones, es necesario evaluar en cada caso concreto y elegir realizar la programación mediante alguna de las estrategias de resolución presentadas. En el caso de que esta probabilidad sea baja para determinado año, es conveniente el modelo de ambas rondas, ya que se obtiene el óptimo global para ambas rondas. Otro aspecto importante también, es que permite encontrar factibilidad para ambas, lo que al programar solo la ronda uno con su modelo correspondiente no asegura factibilidad para la ronda 2 al buscar el óptimo local de esta. A pesar de que para las circunstancias del concurso 2019/2020 no utilizamos la metodología alternativa de resolución por fase, es importante aclarar que en los próximos años puede resultar útil.

Consideramos que a futuro se podría adaptar y generalizar nuestro modelo para que sea útil e independiente de la cantidad y clasificación de las agrupaciones e incluso para otros concursos con características similares. También, esperamos que nuestra investigación sea fuente de motivación para otros que deseen involucrarse en la planificación de eventos ya sea de índole deportiva, académica o artística. Una modificación al proyecto podría ser cambiar el enfoque por uno que procure maximizar la concurrencia del público. Esto implicaría trabajar en conjunto con otras áreas 
como marketing o comunicación. Por otro lado, para modelar la realidad se podría ponderar los diferentes horarios y días, ya que la concurrencia de público depende de los mismos. De lo contrario, podría también evaluarse otras formas de medir la concurrencia. Por último, con el objetivo de reducir el tiempo de procesamiento para resolver ambas rondas, se podría investigar el uso otras metodologías de resolución, como por ejemplo mediante el desarrollo de procedimientos heurísticos basados en alguna metaheurística. 


\section{Referencias}

[1] F. Ortega, M. Pozo, J. Puerto. Modelling and planning public cultural schedules for efficient use of resources. Computers \& Operations Research 58, 9-23, 2015.

[2] Uno por uno, los 10 líos que dejó este carnaval. Diario El Observador.

https://www.elobservador.com.uy/nota/uno-por-uno-los-10-lios-que-dejo-este-carnaval-

201936155957. Último acceso: 14/02/2021

[3] Real Academia Española. Diccionario de la lengua española (Edición de tricentenario), 2008.

[4] G. Kendall, S. Knust, C. Ribeiro, S. Urrutia. Scheduling in sports: An annotated bibliography. Computers \& Operations Research 37, 1-19, 2010.

[5] Boletín Cultura. Sección: Reglamentos del Carnaval 2020.

http://www.boletincultura.com/2019/11/reglamentos-del-carnaval-2020.html. Último acceso:

$15 / 12 / 2019$

[6] Equilibrio y taquilla: estudiantes de ingeniería diseñaron el fixture del carnaval de las promesas. Diario El Observador. https://www.elobservador.com.uy/nota/equilibrio-y-taquilla-estudiantesde-ingenieria-disenaron-el-fixture-del-carnaval-de-las-promesas-2019112810611. Último acceso: $14 / 02 / 2021$ 